\title{
Induction of autophagy contributes to the myocardial protection of valsartan against ischemia-reperfusion injury
}

\author{
XIAOQIAN WU*, LISHAN HE*, YI CAI, GUIPING ZHANG, YULIAN HE, \\ ZHIJIA ZHANG, XIAOEN HE, YANHUA HE, GENSHUI ZHANG and JIANDONG LUO
}

Department of Pharmacology, Guangzhou Medical University, Guangzhou, Guangdong 510182, P.R. China

Received June 21, 2013; Accepted September 20, 2013

DOI: $10.3892 / \mathrm{mmr} .2013 .1708$

\begin{abstract}
The mechanisms underlying the myocardial protection of valsartan against ischemia/reperfusion (I/R) injury are complicated and remain unclear. The aim of this study was to investigate whether autophagy machinery was involved in the protection against I/R injury that is induced by valsartan. In vivo rat hearts were subjected to ischemia by $30 \mathrm{~min}$ ligation of the left anterior descending coronary artery, followed by a 120 min reperfusion. 3-methyladenine (3-MA), a specific inhibitor on autophagic sequestration, was used to inhibit autophagy. The hemodynamics, infarct size of the ventricle and LC3B protein were measured. Western blot analysis was performed to investigate the mechanism by which autophagy was induced by valsartan. Valsartan preconditioning resulted in a significant decrease in infarct size and induced autophagy in the rat heart subjected to I/R injury. The hemodynamics assay showed that the valsartan-induced cardiac functional recovery was attenuated by 3-MA. By contrast, 3-MA decreased the improvement induced by valsartan on the histology and infarction of the rat heart subjected to I/R injury. Valsartan preconditioning induced autophagy via the AKT/mTOR/S6K pathway, independent of Beclin1. In conclusion, valsartan preconditioning induced autophagy via the AKT/mTOR/S6K pathway, which contributed to the myocardial protection against I/R injury.
\end{abstract}

Correspondence to: Dr Xiaoqian Wu, Dr Genshui Zhang or Dr Jiandong Luo, Department of Pharmacology, Guangzhou Medical University, 195 Dongfengxi Road, Guangzhou, Guangdong 510182, P.R. China

E-mail: wuxiaoqian2004@hotmail.com

E-mail: zhanggs99@hotmail.com

E-mail: jiandongluo@hotmail.com

*Contributed equally

Key words: autophagy, valsartan, ischemia/reperfusion injury, AKT, mTOR

\section{Introduction}

Ischemic heart disease is commonly associated with acute blockage of the coronary artery and remains one of the leading causes of morbidity and mortality worldwide. Rapid reperfusion is critical to salvage viable myocardium following coronary occlusion. This reperfusion is commonly achieved either by thrombolysis or percutaneous coronary intervention. However, reperfusion itself may result in cardiac dysfunction termed myocardial ischemia/reperfusion injury (MI/RI), which has been shown to affect clinical outcomes. Therefore, it is essential to investigate the mechanisms of MI/RI to elucidate potential prevention strategies (1).

Angiotensin receptor blockers (ARBs) have yielded encouraging results in animal models and a few have been tested in humans, however, the mechanism(s) of action associated with MI/RI remain unclear. Previous clinical and experimental studies have suggested that the cardio-protective effects of ARBs may extend to the mechanisms beyond lowering blood pressure, including anti-inflammation, anti-atherosclerosis and target organ protection $(2,3)$. It has been previously reported that valsartan may protect the heart from acute $I / R$ via inhibition of the toll-like receptor 4 (TLR4)/nuclear factor (NF)- $\mathrm{B}$ pathway and inflammation (4). Sawicki et al (5) reported that valsartan improved the balance between tissue inhibitor of metalloproteinase (TIMP)-3 and matrix metalloproteinase (MMP)-9, which attenuated the cardiac remodeling following myocardial I/R injury. Iekushi et al (6) demonstrated that valsartan significantly attenuated an increase in the levels of periostin, an extracellular matrix protein, which is important in left ventricular remodeling. The inhibition of periostin by valsartan may contribute to its cardioprotection against myocardial infarction. These studies suggested that the ARBs may exert cardiac protection via a number of potential mechanism(s), independent of blood pressure lowering, which remain unclear.

Autophagy is a highly conserved anabolic process and is implicated as a cell survival/death mechanism, it degrades and recycles long-lived cytoplasmic proteins and organelles to maintain cellular homeostasis and allow adaptation to nutrient depletion $(7,8)$. Autophagy is recognized to be an important process that may be a key regulator of ischemic heart disease; therefore, the involvement of autophagy in myocardial infarction is being investigated (9-13). Inefficient autophagy or its absence, results in poor performance of the myocardium and 
inhibition of starvation-induced autophagy results in cardiac dysfunction and dilatation $(9,10)$. Moreover, autophagy has been shown to be an adaptive response of the heart that protects the myocardium from homodynamic overload and acute ischemic mortality $(11,12)$. In addition, it has been previously reported that Chloramphenicol succinate induces autophagy and protects the swine heart from I/R injury (13), suggesting that autophagy facilitates cardiomyocyte survival as a protective mechanism. In the current study, it was investigated whether autophagy is involved in valsartan-induced myocardioprotection in the rat acute $\mathrm{I} / \mathrm{R}$ model.

\section{Materials and methods}

Animals and drug preconditioning. Male Sprague-Dawley rats (weight, 220-250 g) were purchased from the Medical Experimental Animal Center of Guangdong Province (Guangzhou, China). The investigation conformed with the Guide for the Care and Use of Laboratory Animals published by the United States National Institutes of Health (NIH publication no. 85-23, revised 1996). The study was approved by the Animal Research Committee, Guangzhou Medical University (Guangzhou, China). The rats were randomly divided into four groups: (i) Sham (sham, $n=8$ ); (ii) control with ischemia/reperfusion surgery ( $/ \mathrm{R}, \mathrm{n}=12)$; (iii) $\mathrm{I} / \mathrm{R}$ plus valsartan preconditioning $(\mathrm{I} / \mathrm{R}+\mathrm{Val}, \mathrm{n}=12)$; (iv) $\mathrm{I} / \mathrm{R}$ plus valsartan preconditioning plus $3 \mathrm{MA}(\mathrm{I} / \mathrm{R}+\mathrm{Val}+3 \mathrm{MA}, \mathrm{n}=12)$; and (v) sham plus 3MA preconditioning (Sham+3MA, $n=8)$. Valsartan was supplied by Beijing Novartis Pharma Ltd. (Beijing, China) and dissolved in distilled water on the day of administration. The valsartan suspension $(30 \mathrm{mg} / \mathrm{kg} /$ day) was administered intraperitoneally for seven days continuously prior to I/R surgery. 3MA was purchased from Sigma-Aldrich (St. Louis, MO, USA) and was administered once $30 \mathrm{~min}$ prior to left anterior descending coronary artery (LAD) ligation from the external jugular vein at a dose of $1 \mathrm{mg} / \mathrm{kg}$.

Myocardial I/R surgery. Myocardial I/R was achieved by temporarily occluding the LAD and then releasing the occlusion, similar to the method described previously (14). Briefly, the rats were anesthetized with pentobarbital $(30 \mathrm{mg} / \mathrm{kg}$ intraperitoneally) and the LAD was ligated using a 6-0 silk suture (Ningbo Medical Needle Co., Ltd., Ningbo, China). The electrocardiogram (Fujifilm VisuaSonics, Inc., Toronto, ON, Canada) was monitored for changes in the ST-T segment resulting from tightening or loosing of the ligature. Following $30 \mathrm{~min}$ ischemia, the myocardium was reperfused by restoring the coronary circulation for $2 \mathrm{~h}$. The sham control group underwent the same procedures, with the exception of LAD ligation.

Heart functional measurements. A catheter (1.4F SPR-671, Millar Instruments, Houston, TX, USA) was connected to a pressure transducer (Power Lab, AD Instruments, Colorado Springs, CO, USA) and was directly inserted into the left ventricle (LV) to measure left ventricular systolic pressure (LVSP), left ventricular end-diastolic pressure (LVEDP) and heart rate $(\mathrm{HR})$. The maximum increase rate of left ventricular pressure $(+\mathrm{dP} / \mathrm{dt})$ max and maximum decrease rate of left ventricular pressure $(-\mathrm{dP} / \mathrm{dt}) \mathrm{max}$ were directly calculated from the selected stable recordings.
Determination of infarct size. Upon completion of the experiment, the ventricles were collected and sliced transversely into 2 -mm-thick slices. The slices were incubated in $1 \%$ 2,3,5-triphenyl tetrazolium chloride (TTC; $\mathrm{pH}$ 7.4) for $20 \mathrm{~min}$ at $37^{\circ} \mathrm{C}$. The infarcted area was shown as the area unstained by TTC, measured by Image-Pro Plus 5.0 (Media Cybernetics Inc., Rockville, MD, USA). Infarct size was expressed as a percentage of left ventricular volume $(\%=$ infarct size/left ventricle area).

Histological examination. Specimens of the ventricle tissue were fixed, sectioned and stained with hematoxylin and eosin (H\&E) as described previously (15).

Electron microscopy. Fractions $\left(1 \mathrm{~mm}^{3}\right)$ from fresh ventricles were pre-fixed in a solution of $2.5 \%$ glutaraldehyde and $1 \%$ osmium tetroxide, post-fixed in $1 \%$ osmium tetroxide, dehydrated in an ascending series of alcohols and embedded in epoxy resin. Ultrathin sections were stained with uranyl acetate and lead citrate. Samples were viewed under a transmission electron microscope (H-600; HITACHI, Tokyo, Japan) and analyzed using the IBAS 2.0 Image Analysis system (Kontron, Deggendorf, German).

Western blot analysis. Western blotting was performed as described previously (16). The primary antibodies used were as follows: Anti-LC3B (1:1000; ab51520; Abcam, Cambridge, MA, USA), anti Beclin 1 (1:1000; \#3738), anti-Phospho-AKT ser473 (1:1000; \#4060), anti-AKT (1:1000; \#9272), anti-phospho-mTOR ser2448 (1:1000; \#5536) anti-mTOR (1:1000; \#2972), anti-phospho-4E-BP1 thr37/46 (1:1000; \#2855), anti-4E-BP1 (1:1000; \#9644) and anti- $\beta$ actin (1:1000) (Cell Signaling Technology, Inc., Danvers, MA, USA). The intensity of protein bands was analyzed by Labworks software (ADInstruments Pty Ltd., Bella Vista, Australia).

Statistical analysis. All data were analyzed with the statistical software GraphPad Prism 5.0 (GraphPad Software, Inc., La Jolla, CA, USA) and are expressed as the mean \pm SEM. The differences between two groups were analyzed using Student's unpaired t-test and differences between three or more groups were evaluated using a one-way analysis of variance with Bonferroni correction. $\mathrm{P}<0.05$ was considered to indicate a statistically significant difference.

\section{Results}

Valsartan preconditioning improves cardiac hemodynamics and decreases infarct size in rats subjected to I/R injury. It has been previously shown that valsartan treatment may protect against I/R injury in isolated rat hearts (4,5). In the current study, the ability of valsartan to protect the heart against I/R injury in an in vivo rat model was investigated. The left coronary artery was occluded for $30 \mathrm{~min}$ followed by $2 \mathrm{~h}$ of reperfusion. As shown in Table I, valsartan pretreatment significantly improved the functional recovery with a markedly improved HR, LVSP and $\pm \mathrm{dP} / \mathrm{dtmax}$ compared with the I/R group at the different time points of reperfusion. No infarction was detected in the sham hearts. Compared with control rats in the I/R group, valsartan preconditioning limited the infarct size significantly 
Table I. Cardiac hemodynamics in rats subjected to ischemia-reperfusion.

A, Baseline

\begin{tabular}{|c|c|c|c|c|c|}
\hline & $\begin{array}{l}\text { LVSP, } \\
\mathrm{mmHg}\end{array}$ & $\begin{array}{l}\text { LVEDP, } \\
\text { mmHg }\end{array}$ & $\begin{array}{c}(+\mathrm{dp} / \mathrm{dt}) \max \\
\mathrm{mmHg} / \mathrm{sec}\end{array}$ & $\begin{array}{c}(-\mathrm{dp} / \mathrm{dt}) \max \\
\mathrm{mmHg} / \mathrm{sec}\end{array}$ & $\begin{array}{c}\mathrm{HR}, \\
\text { beats/min }\end{array}$ \\
\hline \multicolumn{6}{|l|}{ Basline } \\
\hline Sham & $134 \pm 3$ & $19 \pm 5$ & $6513 \pm 556$ & $-5708 \pm 450$ & $435 \pm 22$ \\
\hline $\mathrm{I} / \mathrm{R}$ & $129 \pm 4$ & $19 \pm 3$ & $6114 \pm 530$ & $-5174 \pm 318$ & $408 \pm 12$ \\
\hline $\mathrm{I} / \mathrm{R}+\mathrm{Val}$ & $129 \pm 5$ & $18 \pm 2$ & $6038 \pm 540$ & $-5364 \pm 314$ & $422 \pm 6$ \\
\hline $\mathrm{I} / \mathrm{R}+\mathrm{Val}+3 \mathrm{MA}$ & $129 \pm 7$ & $16 \pm 2$ & $6060 \pm 186$ & $-5559 \pm 326$ & $413 \pm 19$ \\
\hline Sham+3MA & $130 \pm 4$ & $18 \pm 4$ & $6421 \pm 645$ & $-5629 \pm 338$ & $421 \pm 20$ \\
\hline
\end{tabular}

B, Reperfusion

$\begin{array}{ccccc}\text { LVSP, } & \text { LVEDP, } & (+\mathrm{dp} / \mathrm{dt}) \mathrm{max}, & (-\mathrm{dp} / \mathrm{dt}) \mathrm{max}, & \mathrm{HR}, \\ \mathrm{mmHg} & \mathrm{mmHg} & \mathrm{mmHg} / \mathrm{sec} & \mathrm{mmg} / \mathrm{sec} & \text { beats/min }\end{array}$

\begin{tabular}{|c|c|c|c|c|c|}
\hline \multicolumn{6}{|l|}{$0 \mathrm{~min}$} \\
\hline Sham & $135 \pm 5$ & $16 \pm 3$ & $6026 \pm 577$ & $-4917 \pm 501$ & $435 \pm 25$ \\
\hline $\mathrm{I} / \mathrm{R}$ & $102 \pm 4$ & $19 \pm 2$ & $3929 \pm 474$ & $-3346 \pm 334$ & $336 \pm 26$ \\
\hline $\mathrm{I} / \mathrm{R}+\mathrm{Val}$ & $124 \pm 4^{\mathrm{a}}$ & $16 \pm 2$ & $5269 \pm 336^{\mathrm{a}}$ & $-3822 \pm 231$ & $436 \pm 16^{a}$ \\
\hline $\mathrm{I} / \mathrm{R}+\mathrm{Val}+3 \mathrm{MA}$ & $111 \pm 2^{\mathrm{b}}$ & $16 \pm 2$ & $5208 \pm 116$ & $-3990 \pm 137$ & $421 \pm 14$ \\
\hline Sham+3MA & $129 \pm 4$ & $17 \pm 3$ & $6083 \pm 494$ & $-4823 \pm 459$ & $428 \pm 22$ \\
\hline \multicolumn{6}{|l|}{$30 \mathrm{~min}$} \\
\hline Sham & $134 \pm 8$ & $13 \pm 2$ & $5860 \pm 637$ & $-4782 \pm 536$ & $415 \pm 19$ \\
\hline $\mathrm{I} / \mathrm{R}$ & $100 \pm 5$ & $20 \pm 2$ & $3637 \pm 349$ & $-2557 \pm 287$ & $305 \pm 27$ \\
\hline $\mathrm{I} / \mathrm{R}+\mathrm{Val}$ & $119 \pm 4^{\mathrm{a}}$ & $13 \pm 2^{\mathrm{a}}$ & $5376 \pm 266^{\mathrm{a}}$ & $-4019 \pm 213^{a}$ & $419 \pm 15^{\mathrm{a}}$ \\
\hline $\mathrm{I} / \mathrm{R}+\mathrm{Val}+3 \mathrm{MA}$ & $116 \pm 4$ & $16 \pm 1$ & $3554 \pm 456^{\mathrm{b}}$ & $-3230 \pm 107$ & $409 \pm 13$ \\
\hline Sham+3MA & $129 \pm 7$ & $15 \pm 2$ & $5689 \pm 567$ & $-4682 \pm 521$ & $422 \pm 20$ \\
\hline \multicolumn{6}{|l|}{$60 \mathrm{~min}$} \\
\hline Sham & $124 \pm 7$ & $14 \pm 2$ & $5739 \pm 472$ & $-4548 \pm 477$ & $391 \pm 17$ \\
\hline $\mathrm{I} / \mathrm{R}$ & $110 \pm 4$ & $20 \pm 2$ & $3712 \pm 323$ & $-2748 \pm 326$ & $290 \pm 32$ \\
\hline $\mathrm{I} / \mathrm{R}+\mathrm{Val}$ & $121 \pm 5^{\mathrm{a}}$ & $13 \pm 1^{\mathrm{a}}$ & $5099 \pm 264^{\mathrm{a}}$ & $-3726 \pm 236^{a}$ & $414 \pm 15^{\mathrm{a}}$ \\
\hline $\mathrm{I} / \mathrm{R}+\mathrm{Val}+3 \mathrm{MA}$ & $103 \pm 5^{\mathrm{b}}$ & $16 \pm 1$ & $3598 \pm 410^{\mathrm{b}}$ & $-3037 \pm 119$ & $369 \pm 10^{b}$ \\
\hline Sham+3MA & $127 \pm 7$ & $15 \pm 3$ & $5690 \pm 452$ & $-4620 \pm 521$ & $417 \pm 18$ \\
\hline \multicolumn{6}{|l|}{$120 \mathrm{~min}$} \\
\hline Sham & $118 \pm 5$ & $14 \pm 1$ & $4926 \pm 146$ & $-4127 \pm 183$ & $397 \pm 23$ \\
\hline $\mathrm{I} / \mathrm{R}$ & $92 \pm 7$ & $19 \pm 1$ & $3316 \pm 341$ & $-2446 \pm 288$ & $284 \pm 37$ \\
\hline $\mathrm{I} / \mathrm{R}+\mathrm{Val}$ & $112 \pm 4^{\mathrm{a}}$ & $11 \pm 1^{\mathrm{a}}$ & $4495 \pm 211^{\mathrm{a}}$ & $-3792 \pm 181^{\mathrm{a}}$ & $393 \pm 18^{a}$ \\
\hline $\mathrm{I} / \mathrm{R}+\mathrm{Val}+3 \mathrm{MA}$ & $94 \pm 5^{\mathrm{b}}$ & $16 \pm 2^{b}$ & $3030 \pm 114^{b}$ & $-2817 \pm 840^{b}$ & $343 \pm 14^{b}$ \\
\hline Sham+3MA & $124 \pm 5$ & $15 \pm 3$ & $5243 \pm 325$ & $-4512 \pm 367$ & $402 \pm 20$ \\
\hline
\end{tabular}

The parameters of LVSP, LVEDP and HR prior to ischemia (baseline) and at different times of reperfusion in the sham, I/R, I/R+Val, $\mathrm{I} / \mathrm{R}+\mathrm{Val}+3 \mathrm{MA}$ and Sham+3MA groups. Values are presented as the mean $\pm \mathrm{SEM}$. ${ }^{\mathrm{a}} \mathrm{P}<0.05$, vs. I/R $(\mathrm{n}=8)$ and ${ }^{\mathrm{b}} \mathrm{P}<0.05$, vs $\mathrm{I} / \mathrm{R}+\mathrm{Val}(\mathrm{n}=8)$. LVSP, left ventricular systolic pressure; LVEDP, left ventricular end-diastolic pressure; HR, heart rate; 3-MA, 3-methyladenine; I/R, ischemia/reperfusion group; I/R+Va, I/R plus Valsartan preconditioning group; I/R+Val+3MA, I/R+Va 3MA group; Sham+3MA, sham plus 3MA group.

as shown in Fig. 1. These data demonstrated that valsartan pretreatment protects the heart against $\mathrm{I} / \mathrm{R}$ injury in vivo.

Valsartan preconditioning induces autophagy in the rat hearts subjected to I/R injury. To investigate the molecular mechanisms by which valsartan protects the rat heart against I/R injury, the potential involvement of prosurvival autophagy was observed. The electrographic assay showed that autophagic vacuoles were significantly increased in the rats of the I/R+Val group compared with the I/R group (Fig. 2A). The conversion of LC3-I to LC3-II was used as a specific marker of autophagy. As shown in Fig. 2B, valsartan preconditioning significantly increased the ratio of LC3-II/LC3-I proteins in the hearts of the I/R+Val group compared with I/R alone. These data suggest 
A

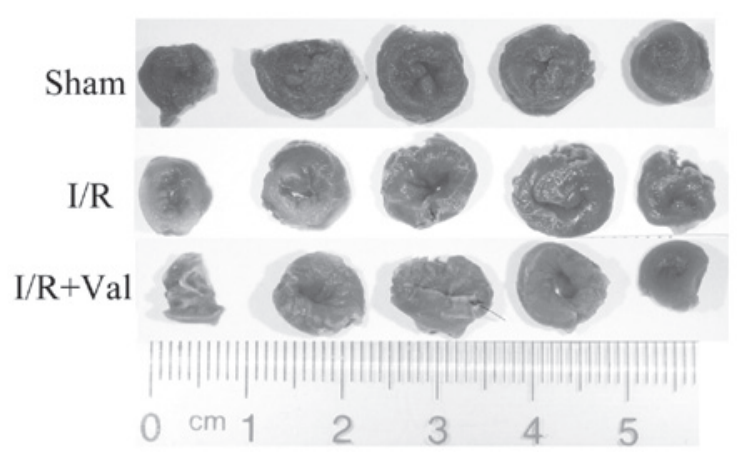

B

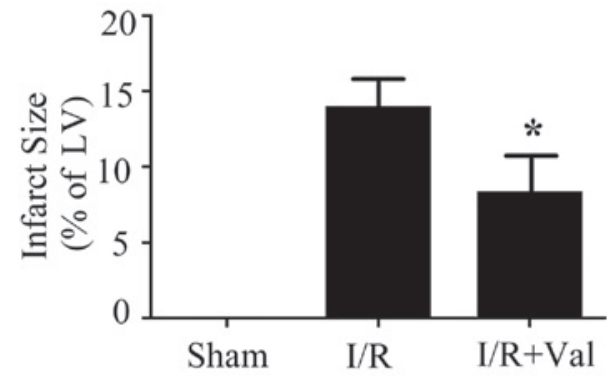

Figure 1. Val preconditioning decreased infarct size in the rats subjected to I/R injury. The infarcted area is shown as the area unstained by $2,3,5$-triphenyl tetrazolium chloride, measured by the Image-Pro Plus 5.0 (A and B). Data are shown as the representative results from four mice in the sham, I/R and I/R+Val groups. Values are presented as the mean \pm SEM. "P<0.05, vs. I/R. I/R, ischemia/reperfusion; Val, Valsartan; LV, left ventricle.
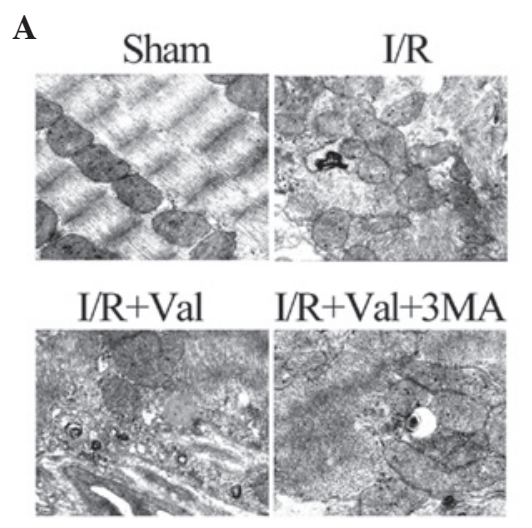

B
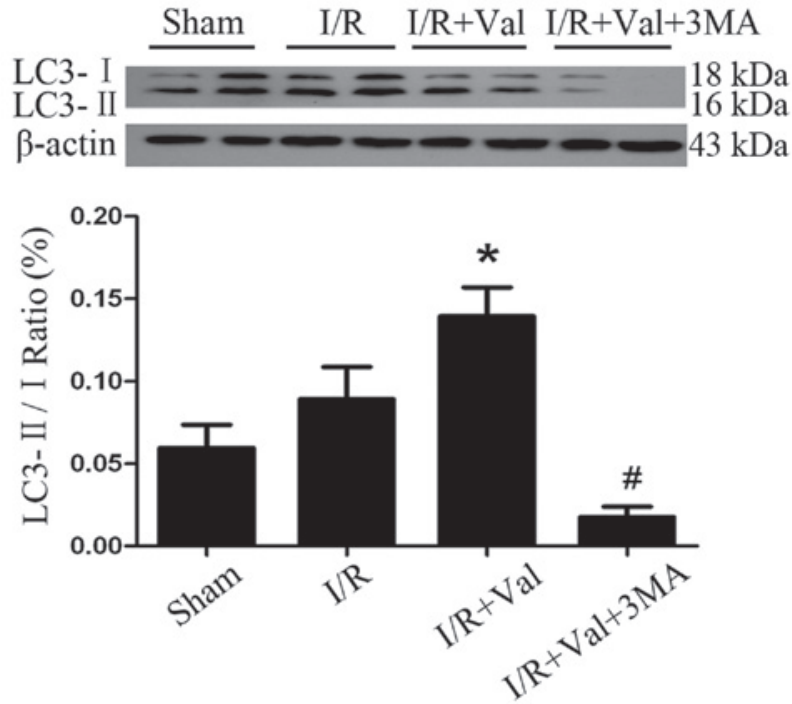

Figure 2. Valsartan preconditioning induced autophagy in the rat hearts subjected to I/R injury. (A) Electron micrographs (magnification, $x 20,000)$ of the infarct area in the sham, I/R, I/R+Val and I/R+Val+3MA groups. (B) Western blot analysis of the autophagy-associated protein, LC3, in the ventricle tissue of the aforementioned groups. Values are presented as the mean \pm SEM. ${ }^{*} \mathrm{P}<0.05$, vs. I/R and ${ }^{\#} \mathrm{P}<0.05$, vs. I/R+Val. I/R, ischemia/reperfusion; Val, Valsartan; 3-MA, 3-methyladenine.
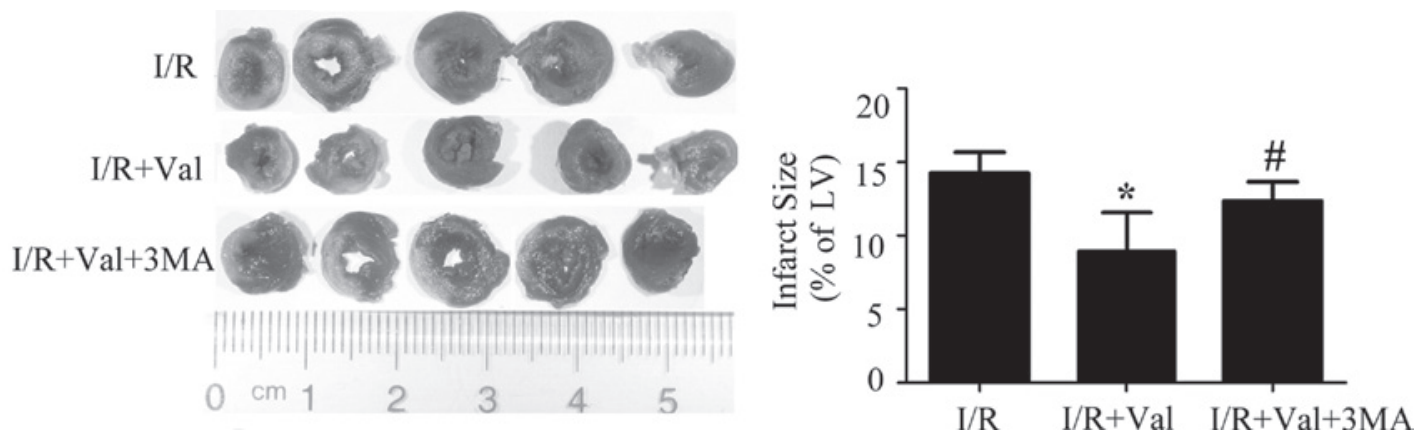

Figure 3. 3MA eliminated the improvement of Val on infarct size of the hearts subjected to I/R injury. The infarcted area was shown as the area unstained by 2,3,5-triphenyl tetrazolium chloride, measured by Image-Pro Plus 5.0. Data are shown as the representative results from four mice in the groups as indicated. Values are presented as the mean $\pm \mathrm{SEM}$. ${ }^{*} \mathrm{P}<0.05$, vs. I/R and ${ }^{\text {" }} \mathrm{P}<0.05$, vs. I/R+Val. I/R, ischemia/reperfusion; Val, Valsartan; 3-MA, 3-methyladenine.

that valsartan preconditioning induced autophagy activation in the rat hearts subjected to I/R injury.

Autophagy inhibition weakens the effect of valsartan on the improvement of hemodynamics and histology in the rats subjected to I/R injury. To investigate the potential involvement of prosurvival autophagy in valsartan-induced cardioprotection, 3MA, a specific inhibitor of autophagy, was administrated intravenously on the day of surgery, 30 min prior to LAD ligation and following valsartan preconditioning. The hemodynamic 


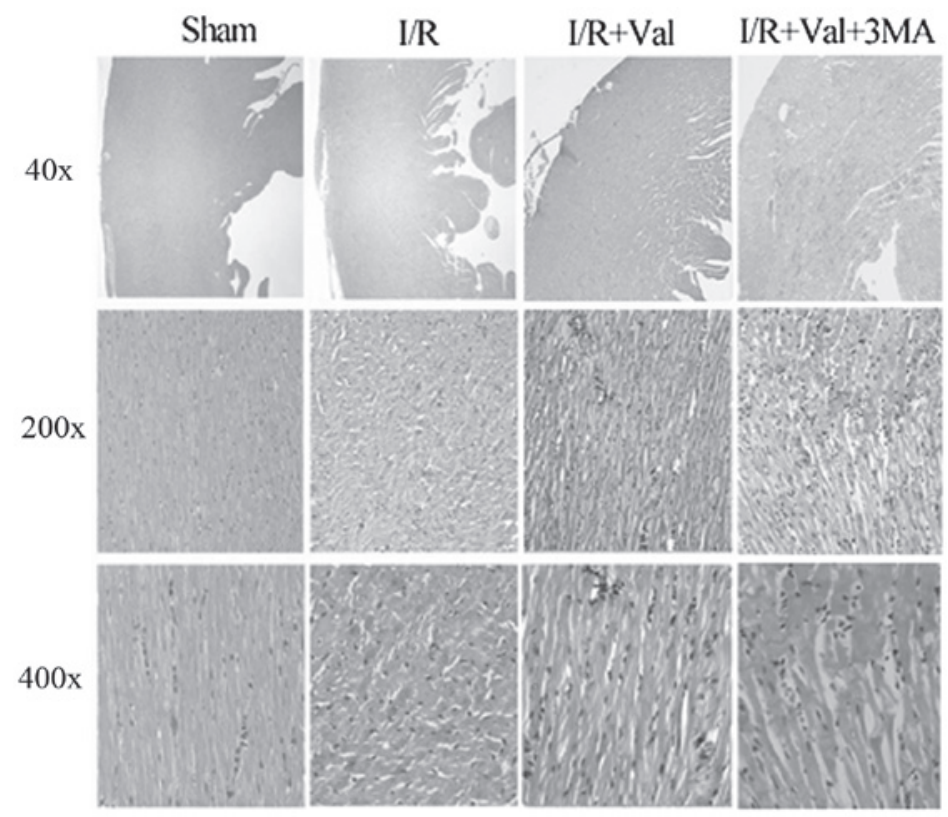

Figure 4. 3MA eliminated the improvement of Val on histology of the hearts subjected to I/R injury. Hematoxylin and eosin staining of rats myocardium in the groups as indicated. I/R, ischemia/reperfusion; Val, Valsartan; 3-MA, 3-methyladenine.

A

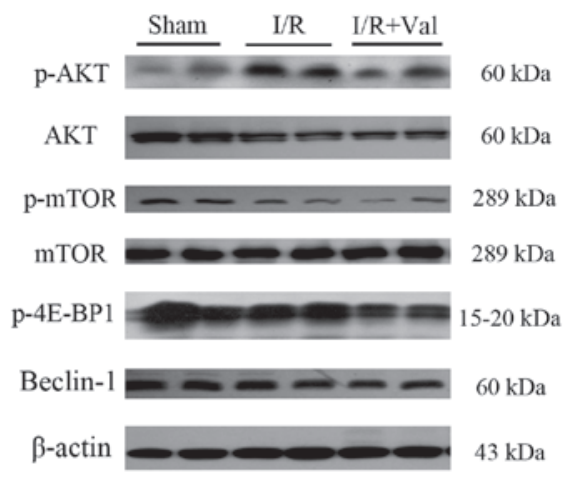

C

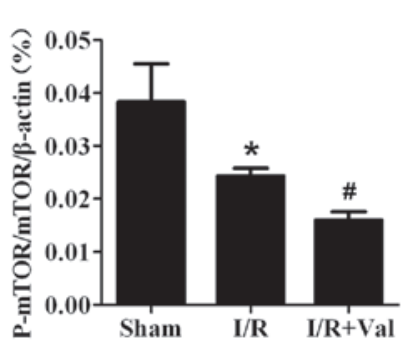

D

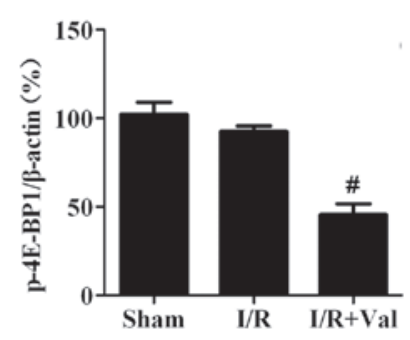

B

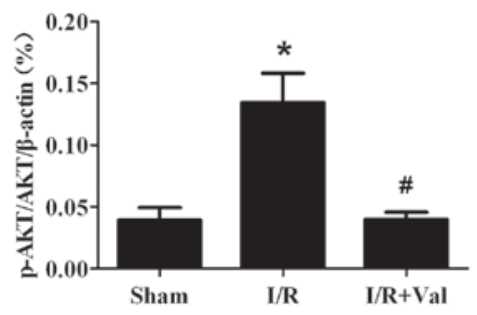

$\mathbf{E}$

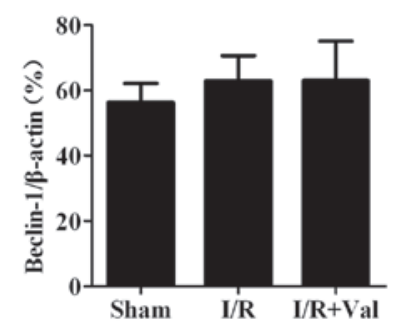

Figure 5. Valsartan induced autophagy via the AKT/mTOR/S6K signaling pathway. (A) Western blot analysis of Akt, mTOR, 4E-BP and Beclin-1 phosphorylation and total protein in the ventricle tissue of the groups as indicated. Data are shown as the representative results from six mice per group and quantified using the Image J software (mean $\pm \mathrm{SEM}$; ${ }^{*} \mathrm{P}<0.05$, vs. $\mathrm{I} / \mathrm{R}$ and ${ }^{\#} \mathrm{P}<0.05$, vs. $\mathrm{I} / \mathrm{R}+\mathrm{Val}$ ) for different proteins as indicated in B-E.

assessment showed that the parameters of cardiac function were significantly attenuated by the $3 \mathrm{MA}$ pre-treatment at the $120 \mathrm{~min}$ reperfusion time point in the $\mathrm{I} / \mathrm{R}+\mathrm{Val}+3 \mathrm{MA}$ rats compared with the I/R+Val group $(\mathrm{P}<0.05, \mathrm{n}=8$; Table I).

As shown in Fig. 3, the infarct size in the rats of the $\mathrm{I} / \mathrm{R}+\mathrm{Val}+3 \mathrm{MA}$ group significantly increased compared with that of the $\mathrm{I} / \mathrm{R}+$ Val group $(12.35 \pm 1.32$ vs. $9.17 \pm 4.68 \%, \mathrm{P}<0.05$, $\mathrm{n}=6)$. $H \& E$ staining revealed that valsartan pretreatment significantly improved the cardiac morphology compared with the I/R control and this effect was eliminated by 3-MA
(Fig. 4). Cardiac muscle fibers were lined up in order and transverse striation was clear and well-distributed in the rats of the sham group. In the heart sections of the rats subjected to I/R injury, cardiomyocyte damage was characterized by wavy fibers, irregular transverse striation and edema with neutrophil infiltration, which was attenuated by valsartan pretreatment. Notably, the hearts of rats in the I/R+Val+3MA group exhibited increased injury compared with those of the $\mathrm{I} / \mathrm{R}+\mathrm{Val}$ group. These data suggested that valsartan-mediated cardiac protection was weakened by 3-MA. 
Valsartan induces autophagy via the AKT/mTOR/S6K signaling pathway. To corroborate the mechanism by which valsartan pretreatment induced autophagy, western blot analysis was performed to examine the two most well-understood pathways that regulate autophagy, the AKT/mTOR and Beclin-1 pathways. As shown in Fig. 5, AKT was phosphorylated in the hearts of $\mathrm{I} / \mathrm{R}$ controls and this effect was reversed with valsartan pretreatment. In addition, phosphorylation of 4E-BP1 was significantly attenuated by valsartan pretreatment compared with I/R only. By contrast, Beclin-1 was not changed. The results indicated that valsartan pretreatment induced autophagy by means of the AKT/mTOR pathway, without modifying Beclin-1 levels.

\section{Discussion}

This study showed that valsartan, administered as a pretreatment, provided resistance for the rat heart against I/R injury. Moreover, the results suggested that myocardial protection of valsartan is associated with increased autophagy. The underlying mechanism of valsartan-induced autophagy was observed to occur via the AKT/mTOR signaling pathway.

Autophagy is an essential cellular mechanism that mediates continuous recycling of intracellular components. As a response to stress conditions, such as I/R, autophagy digests cytoplasmic materials to generate essential metabolic substrates and energy to maintain live cells $(8,17)$. Autophagy is recognized to be a potent cell survival mechanism and has been shown to be involved in the ischemic tolerance observed in the heart and neurons $(10-12,18,19)$. However, whether autophagy is the key mechanism of the cardiac protection conferred by valsartan preconditioning in heart I/R injuries remains unknown. Therefore, the aim of the current study was to determine whether autophagy mediates the protective effects of valsartan preconditioning in the heart. In the current study: (i) autophagy was observed to be activated in the hearts subjected to I/R injury, which was enhanced by valsartan pretreatment; (ii) the protective effects of valsartan preconditioning were eliminated with autophagy inhibition in vivo. These results revealed that the myocardial protection of valsartan against I/R injury depends, at least in part, on the activation of autophagy. It has been reported that valsartan exerted protection on the heart against I/R injury via an Angiotensin II-independent mechanism (4-6). Iekushi et al (6) showed that a novel mechanism of valsartan on myocardial infarction occurs via inhibition of the antiadhesion molecule, periostin. Two individual studies reported that valsartan protects the heart from acute $\mathrm{I} / \mathrm{R}$ via inhibition of the TLR4/NF- $\kappa \mathrm{B}$ pathway or by improving the balance between TIMP-3/MMP-9 (4,5). To the best of our knowledge, the current study is the first to investigate valsartan induced autophagy, which is associated with its protection against myocardial I/R injury.

The effect and mechanism of autophagy in ischemic disease has been widely investigated, however, it remains unclear and controversial (9-13). The current results indicate that autophagy was upregulated in the rat heart subjected to I/R injury, which was enhanced by valsartan preconditioning. Matsui et al (20) reported that autophagy was implicated in cell death with the increased infarct size upon reperfusion of the ischemic myocardium. By contrast, it was previously reported that Chloramphenicol succinate induced autophagy and protected the swine heart from I/R injury (13), suggesting that autophagy exerts a protective mechanism against I/R injury. Ma et al (10) reported that I/R injury upregulated cardiomyocyte autophagy as a stress-response mechanism; however, the autophagosome clearance is impaired. This study explained why autophagy has different roles in I/R. In the present study the results indicated that valsartan-induced autophagy exerted protection against myocardial I/R injury.

The current results indicate that valsartan induced autophagy, at least in part, through the suppression of the AKT/mTOR/p70S6K signaling pathway but not Beclin-1. Consistent with the present study, Kondo et al $(21,22)$ have shown that inhibition of the AKT/mTOR/p70S6K pathway by curcumin led to the induction of autophagy in malignant gliomas. Autophagy activation is complex and is not completely understood, and leads to the activation of a wide range of signaling pathways (23-26). The best understood pathway for the regulation of autophagy is the target of rapamycin complex 1 (TORC1), which directly phosphorylates and negatively regulates the Atg1 complex $(23,24,27,28)$. TORC1 acts as a central component of a number stress pathways, linking them to the autophagic machinery and its activity may be inferred by the phosphorylation levels of its target protein, ribosomal S6 kinase and eukaryotic translation initiation factor 4E-bingding protein 1. Upstream, TORC activity and a number of stress responses may be regulated via AKT. AKT was phosphorylated in the hearts of I/R controls and this effect was reversed by valsartan pretreatment. In addition, phosphorylation of 4E-BP1, which is downstream of mTOR, was significantly attenuated by valsartan pretreatment compared with I/R alone. Another pathway that positively regulates autophagy is Beclin-1, which is markedly associated with autophagic cell death (29-31). No change of Beclin-1 was observed. Thus, Figure 5 indicated that valsartan was shown to preserve AKT/mTOR/S6K phosphoralation and did not alter the Beclin-1 level. These findings indicated that valsartan-induced autophagy was AKT/mTOR dependent.

In conclusion, the current study demonstrated that preconditioning with the AT1R antagonist, valsartan, protected against I/R injury via autophagy, which is mTOR dependent. Further studies are required to elucidate the effect of valsartan on autophagy.

\section{Acknowledgements}

This study was supported by grants from the National Natural Science Foundation of China (grant no. 81001436 to Dr. X Wu, grant no. 81000353 to Dr. G Zhang and grant no. 81173062 to Dr. J Luo), the Education Administration Research Foundation of Guangdong Province(grant no. LYM10110 to Dr. X Wu) and the Education Administration Research Foundation of Guangzhou City (grant nos. 10A018G and 10A157 to Dr. X Wu).

\section{References}

1. Tritto I, Zuchi C, Vitale S and Ambrosio G: Therapy against reperfusion-induced microvascular injury. Curr Pharm Des 19: 4586-4596, 2013. 
2. Cianchetti S, Del Fiorentino A, Colognato R, Di Stefano R, Franzoni $F$ and Pedrinelli R: Anti-inflammatory and anti-oxidant properties of telmisartan in cultured human umbilical vein endothelial cells. Atherosclerosis 198: 22-28, 2008.

3. Varagic J, Frohlich ED, Susic D, et al: AT1 receptor antagonism attenuates target organ effects of salt excess in SHRs without affecting pressure. Am J Physiol Heart Circ Physiol 294: H853-H858, 2008.

4. Yang J, Jiang H, Yang J, Ding JW, Chen LH, Li S and Zhang XD: Valsartan preconditioning protects against myocardial ischemia-reperfusion injury through TLR4/NF-kappaB signaling pathway. Mol Cell Biochem 330: 39-46, 2009.

5. Sawicki G, Menon V and Jugdutt BI: Improved balance between TIMP-3 and MMP-9 after regional myocardial ischemia-reperfusion during AT1 receptor blockade. J Card Fail 10: 442-449, 2004.

6. Iekushi K, Taniyama Y, Azuma J, et al: Novel mechanisms of valsartan on the treatment of acute myocardial infarction through inhibition of the antiadhesion molecule periostin. Hypertension 49: 1409-1414, 2007

7. Kundu M and Thompson CB: Autophagy: basic principles and relevance to disease. Annu Rev Pathol 3: 427-455, 2008.

8. Levine B and Kroemer G: Autophagy in the pathogenesis of disease. Cell 132: 27-42, 2008

9. Oka T, Hikoso S, Yamaguchi O, et al: Mitochondrial DNA that escapes from autophagy causes inflammation and heart failure. Nature 485: 251-255, 2012.

10. Ma X, Liu H, Foyil SR, et al: Impaired autophagosome clearance contributes to cardiomyocyte death in ischemia/reperfusion injury. Circulation 125: 3170-3181, 2012.

11. Buss SJ, Muenz S, Riffel JH, et al: Beneficial effects of Mammalian target of rapamycin inhibition on left ventricular remodeling after myocardial infarction. J Am Coll Cardiol 54: 2435-2446, 2009.

12. Marin TM, Keith K, Davies B, et al: Rapamycin reverses hypertrophic cardiomyopathy in a mouse model of LEOPARD syndrome-associated PTPN11 mutation. J Clin Invest 121: 1026-1043, 2011

13. Sala-Mercado JA, Wider J, Undyala VV, et al: Profound cardioprotection with chloramphenicol succinate in the swine model of myocardial ischemia-reperfusion injury. Circulation 122: S179-S184, 2010.

14. Zhai P, Sciarretta S, Galeotti J, Volpe M and Sadoshima J: Differential roles of GSK-3 $\beta$ during myocardial ischemia and ischemia/reperfusion. Circ Res 109: 502-511, 2011

15. Wu X, Cheng J, Li P, et al: Mechano-sensitive transcriptional factor Egr-1 regulates insulin-like growth factor-1 receptor expression and contributes to neointima formation in vein grafts. Arterioscler Thromb Vasc Biol 30: 471-476, 2010

16. Wu X, Huang H, Tang F, Le K, Xu S and Liu P: Regulated expression of endothelial lipase in atherosclerosis. Mol Cell Endocrinol 315: 233-238, 2010
17. Mizushima N: Autophagy: process and function. Genes Dev 21: 2861-2873, 2007.

18. Wang P, Guan YF, Du H, Zhai QW, Su DF and Miao CY Induction of autophagy contributes to the neuroprotection of nicotinamide phosphoribosyltransferase in cerebral ischemia. Autophagy 8: 77-87, 2012.

19. Gustafsson AB and Gottlieb RA: Autophagy in ischemic heart disease. Circ Res 104: 150-158, 2009.

20. Matsui Y, Takagi H, Qu X, et al: Distinct roles of autophagy in the heart during ischemia and reperfusion: roles of AMP-activated protein kinase and Beclin 1 in mediating autophagy. Circ Res 100: 914-922, 2007.

21. Aoki H, Takada Y, Kondo S, Sawaya R, Aggarwal BB amd Kondo Y: Evidence that curcumin suppresses the growth of malignant gliomas in vitro and in vivo through induction of autophagy: role of Akt and extracellular signal-regulated kinase signaling pathways. Mol Pharmacol 72: 29-39, 2007.

22. Shinojima N, Yokoyama T, Kondo Y and Kondo S: Roles of the Akt/mTOR/p70S6K and ERK1/2 signaling pathways in curcumin-induced autophagy. Autophagy 3: 635-637, 2007.

23. Ganley IG, Lam du H, Wang J, Ding X, Chen S and Jiang X ULK1.ATG13.FIP200 complex mediates mTOR signaling and is essential for autophagy. J Biol Chem 284: 12297-12305, 2009.

24. Kim J, Kundu M, Viollet B and Guan KL: AMPK and mTOR regulate autophagy through direct phosphorylation of Ulk1. Nat Cell Biol 13: 132-141, 2011.

25. Egan DF, Shackelford DB, Mihaylova MM, et al: Phosphorylation of ULK1 (hATG1) by AMP-activated protein kinase connects energy sensing to mitophagy. Science 331: 456-461, 2011.

26. Stephan JS, Yeh YY, Ramachandran V, Deminoff SJ and Herman PK: The Tor and PKA signaling pathways independently target the Atg1/Atg13 protein kinase complex to control autophagy. Proc Natl Acad Sci USA 106: 17049-17054, 2009.

27. Nicklin P, Bergman P, Zhang B, et al: Bidirectional transport of amino acids regulates mTOR and autophagy. Cell 136: 521-534, 2009.

28. Yu L, McPhee CK, Zheng L, et al: Termination of autophagy and reformation of lysosomes regulated by mTOR. Nature 465: 942-946, 2010

29. Zou M, Lu N, Hu C, et al: Beclin 1-mediated autophagy in hepatocellular carcinoma cells: implication in anticancer efficiency of oroxylin A via inhibition of mTOR signaling. Cell Signal 24: 1722-1732, 2012.

30. Zou Z, Wu L, Ding H, et al: MicroRNA-30a sensitizes tumor cells to cis-platinum via suppressing beclin 1-mediated autophagy. J Biol Chem 287: 4148-4156, 2012.

31. Ugland H, Naderi S, Brech A, Collas P and Blomhoff HK: cAMP induces autophagy via a novel pathway involving ERK, cyclin $\mathrm{E}$ and Beclin 1. Autophagy 7: 1199-1211, 2011. 